\title{
Punção Acidental da Dura e Cefaleia Pós-punção da Dura na População Obstétrica: Oito Anos de Experiência
}

\author{
Accidental Dural Puncture and Post-dural Puncture \\ Headache in the Obstetric Population: Eight Years of \\ Experience
}

Maria Vaz ANTUNES $\square^{1}$, Adriano MOREIRA ${ }^{1}$, Catarina SAMPAIO ${ }^{1}$, Aida FARIA ${ }^{1}$

Acta Med Port 2016 Apr;29(4):268-274 - http://dx.doi.org/10.20344/amp.6815

\section{RESUMO}

Introdução: A punção acidental da dura é uma importante complicação da anestesia regional e a cefaleia pós-punção continua a ser causa de morbilidade na população obstétrica. O objetivo do nosso estudo foi calcular a incidência de punção acidental e cefaleia póspunção no nosso Centro Hospitalar e avaliar a sua abordagem entre os anestesiologistas obstétricos.

Material e Métodos: Realizámos uma auditoria retrospetiva, entre janeiro de 2007 e dezembro de 2014. Revimos as folhas de registo das doentes em que ocorreu punção inadvertida da dura ou cefaleia pós-punção. Excluímos as doentes submetidas a bloqueio subaracnoideu. Utilizámos o SPSS 22.0 no tratamento estatístico dos dados.

Resultados: Obtivémos 18497 bloqueios neuro-axiais e 58 punções acidentais da dura (0,3\%). Após punção detetada, em $71,4 \%$ o cateter epidural foi re-posicionado e $21,4 \%$ tiveram cateteres intra-tecais. Quarenta e cinco $(77,6 \%)$ desenvolveram cefaleia e a instituição de medidas profiláticas ocorreu em $76,1 \%$. O tratamento conservador foi efetuado em todas as doentes. O blood patch epidural foi realizado em $32,8 \%$ com um sucesso de $84,2 \%$.

Discussão: A incidência de cefaleia pós-punção não está relacionada com o tipo de parto ou a inserção do cateter intra-tecal. A recolocação do cateter epidural mantém-se a abordagem de eleição após punção. A instituição de medidas profiláticas é uma prática comum, apesar do baixo grau de eficácia. Realizámos blood patch epidural após falência do tratamento conservador.

Conclusão: A incidência de punção inadvertida e cefaleia pós-punção foi semelhante à da literatura. Apesar de ser uma complicação comum, existe falta de consenso na sua abordagem.

Palavras-chave: Analgesia Epidural; Anestesia Obstétrica; Cefaleia Pós-Punção Dural; Dura-Máter; Placa de Sangue Epidural.

\section{ABSTRACT}

Introduction: Accidental dural puncture is an important complication of regional anesthesia and post-dural puncture headache remains a disable outcome in obstetric population. The aim of our study was to calculate the incidence of accidental puncture and post-puncture headache and evaluate its management among obstetric anesthesiologists.

Material and Methods: We conducted a retrospective audit, between January 2007 and December 2014. We reviewed the record sheets of patients who experienced either accidental puncture or post-puncture headache. We excluded the patients undergoing spinal block. We use the SPSS 22.0 for statistical analyses.

Results: We obtained 18497 neuro-axial blocks and 58 accidental dural punctures $(0.3 \%)$. After detected puncture, in $71.4 \%$ epidural catheter was re-positioned and $21.4 \%$ had intra-thecal catheters. Forty-five $(77.6 \%)$ developed headache and the prophylactic measures were established in $76.1 \%$. Conservative treatment was performed in all patients. The epidural blood patch was performed in $32.8 \%$ with a $84.2 \%$ of success.

Discussion: The incidence of post-dural puncture headache is unrelated to the type of delivery or insertion of intrathecal catheter. The re-placement of the epidural catheter remains the main approach after puncture. The institution of prophylactic measures is a common practice, despite the low level of evidence. We performed epidural blood patch after failure of conservative treatment.

Conclusion: The incidence of accidental dural puncture and post-dural puncture headache was similar to the literature. Despite being a common complication, there remains lack of consensus on its approach.

Keywords: Analgesia, Epidural; Anesthesia, Obstetrical; Blood Patch, Epidural; Dura Mater; Post-Dural Puncture Headache.

\section{INTRODUÇÃO}

A punção acidental da dura-máter (PAD) é uma complicação importante da anestesia do neuroeixo e a cefaleia pós-punção da dura (CPPD) é um outcome adverso na população obstétrica. ${ }^{1}$

As grávidas têm maior risco de desenvolver cefaleia após PAD quando comparadas com outras populações, devido à sua idade mais jovem e género. ${ }^{2} \mathrm{~A}$ cefaleia pós-punção é uma causa de morbilidade importante nas parturientes, que resulta num aumento do tempo de internamento hospitalar e no gasto adicional em investigações complementares e no tratamento necessário. Existem também apresentações clínicas tardias, pelo que se torna importante a sensibilização dos especialistas em Medicina Geral e Familiar para esta complicação da anestesia do neuroeixo.

No Reino Unido, a taxa de punção acidental é inferior a $1 \%$ e até $80 \%$ das parturientes desenvolvem sintomas após PAD. ${ }^{1}$ Em Portugal, estas taxas não são conhecidas e a abordagem clínica após a deteção ou suspeita de PAD varia de acordo com os protocolos institucionais. A implementação de medidas profiláticas para o desenvolvimento de cefaleia ainda é efetuada, apesar do reduzido grau de evidência. Após o desenvolvimento de cefaleia, é efetuado

1. Serviço de Anestesiologia. Centro Hospitalar de São João. Porto. Portugal.

$\square$ Autor correspondente: Maria Vaz Antunes. maria_joao_vaz@hotmail.com

Recebido: 16 de julho de 2015 - Aceite: 02 de novembro de 2015 | Copyright @ Ordem dos Médicos 2016 
tratamento conservador e/ou realizado o blood patch epidural (BPE).

O objetivo do nosso estudo foi calcular a incidência da PAD e da CPPD na população obstétrica do nosso Centro Hospitalar e avaliar a abordagem desta complicação entre os Anestesiologistas Obstétricos.

\section{MATERIAL E MÉTODOS}

Realizámos um estudo retrospetivo, entre janeiro de 2007 e dezembro de 2014, aprovado pela Comissão de Ética do nosso Centro Hospitalar, que é um centro obstétrico de referência terciária, onde foram realizados 21772 partos durante o período do estudo.

Considerámos como critério de inclusão a punção acidental da dura ou a cefaleia pós-punção na analgesia/anestesia epidural ou na pesquisa do espaço epidural durante a técnica combinada do neuroeixo (CNE), dados presentes nos registos de punção acidental da dura/cefaleia pós-punção do Serviço de Anestesiologia. A técnica utilizada na pesquisa do espaço epidural foi a perda de resistência com soro fisiológico com uma agulha de Tuohy $18 \mathrm{G}$ e a abordagem do espaço foi por via mediana. Entenda-se como técnica combinada do neuroeixo o bloqueio epi-raquidiano efetuado com agulha através de agulha, em que o espaço epidural é pesquisado conforme o método previamente descrito e a dura-máter é puncionada com uma agulha ponta de lápis com o calibre 27G. A PAD foi diagnosticada com base na saída de líquido cefalorraquidiano (LCR) pela agulha ou pelo cateter epidural ou após o desenvolvimento de cefaleia diagnosticada de acordo com os critérios internacionais de classificação de cefaleia (critérios ICHD-II). Segundo estes critérios, o doente tem de desenvolver cefaleia até 15 minutos após adotar a posição sentada ou de pé e ocorrer alívio da cefaleia dentro de 15 minutos após adotar a posição de decúbito dorsal. O doente deve ter pelos menos um dos seguintes sintomas associados à cefaleia: rigidez da nuca, zumbido, hipoacúsia, fotofobia ou náusea. A cefaleia tem de ocorrer até 14 dias após punção inadvertida, confirmada ou suspeita, e resolver espontaneamente dentro de uma semana ou dentro de 48 horas após início do tratamento eficaz (geralmente blood patch epidural). ${ }^{3}$

Foram excluídos os casos cuja cefaleia resultou de analgesia ou anestesia subaracnoideia exclusiva e os casos em que se verificaram complicações não relacionadas com a punção acidental.

Realizámos a revisão do processo clínico das grávidas sinalizadas e incluímos como variáveis de estudo a idade, semana de gestação em que ocorreu o evento, número de gestações prévias, patologia associada e respetivo score de acordo com a American Society of Anesthesiologists (ASA), índice de massa corporal (IMC), tipo de parto, tipo de técnica anestésica ou analgésica, posição da doente durante a técnica, nível vertebral da punção, local de saída do líquido cefalorraquidiano (LCR) (agulha versus cateter), colocação de cateter subaracnoideu, instituição de medidas profiláticas após verificação da punção, incidência, início e intensidade da cefaleia avaliada com a escala numérica da dor, sintomas associados, instituição de tratamento conservador, incidência e outcome do BPE e tempo de internamento hospitalar.

\section{Análise estatística}

Efetuámos a análise estatística com o IBM SPSS Statistic versão 22.0. Na análise descritiva, as variáveis categóricas foram apresentadas através de frequências absolutas e relativas e as variáveis contínuas foram apresentadas sob a forma de média e valores máximo e mínimo. A comparação de proporções foi realizada pelo teste de qui quadrado ou teste exato de Fisher, quando adequado. Nas comparações entre variáveis contínuas, utilizámos o teste $t$ de Student para amostras independentes. Nos testes realizados, os valores de prova inferiores a 0,05 foram considerados indicativos de significância estatística. Foram construídos modelos de regressão múltipla, mas não obtivémos resultados estatisticamente significativos (dados não apresentados).

\section{RESULTADOS}

Entre janeiro de 2007 e dezembro de 2014, foram realizados 18497 bloqueios neuro-axiais, sendo 276 bloqueios subaracnoideus, 15110 epidurais e 3111 CNE.

A média de idade das doentes com punção acidental que desenvolveram ou não cefaleia foi de 30,4 anos (20 42 , IC 95\%) com uma média de 38,3 semanas de gestação

Tabela 1 - Características demográficas e tipo de parto das grávidas com punção acidental da dura

\begin{tabular}{lc}
\hline & $\begin{array}{c}\text { PAD } \\
(\mathrm{n}=58)\end{array}$ \\
\hline Idade (anos), mín-máx (média) & $20,0-42,0(30,4)$ \\
Idade gestacional (semanas), mín-máx (média) & $34,0-41,0(38,3)$ \\
Gestações (n), mín-máx (média) & $1,0-7,0(1,8)$ \\
ASA, mín-máx (média) & $1-3(1,47)$ \\
IMC (kg/m²), mín-máx (média) & $19,1-44,9(30,0)$ \\
Tipo de parto, $n(\%)$ & $38,0(65,5)$ \\
\end{tabular}

n: número de doentes; ASA: American Society of Anesthesiologists; IMC: índice de massa corporal; PAD: punção acidental da dura. 
(34 - 41, IC 95\%). O índice de massa corporal médio foi de $30 \mathrm{~kg} / \mathrm{m}^{2}(19,1-44,9$, IC 95\%). A maioria (65,5\%) das mulheres foi submetida a parto vaginal (Tabela 1 ).

A incidência de punção acidental da dura, com ou sem desenvolvimento de cefaleia subsequente, foi de 0,3\% (58 em 18 221), em que $72,4 \%$ das punções foram observadas (42 em 58) e em 27,6\% (16 em 58) ocorreu cefaleia após punção acidental não reconhecida. A punção acidental (observada e não reconhecida) ocorreu em 89,7\% (52 em 58) durante a técnica epidural e em 10,3\% (6 em 58) durante a técnica CNE. Neste último subgrupo de doentes (6 em 58), após a punção acidental da dura com a agulha epidural, foi realizada uma técnica combinada agulha através de agulha no espaço vertebral adjacente. A posição de decúbito lateral esquerdo foi utilizada em 69,9\% (40 em 58) das doentes. Em mais de metade das doentes $(58,6 \%)$, a saída do LCR ocorreu através da agulha epidural, incluindo todas as doentes submetidas a técnica CNE (Tabela 2).

Após deteção da punção acidental da dura, em 50,0\% (21 em 42) das grávidas, o cateter epidural foi re-colocado noutro espaço lombar, em 21,4\% (9 em 42) no mesmo espaço lombar, em $21,4 \%$ (9 em 42) o cateter foi introduzido no espaço intra-tecal durante 24 horas e em 7,2\% (3 em 42) foi abandonada a técnica regional. Em 76,1\% (35 em 46) das doentes com PAD detetada ou não reconhecida foram instituídas medidas profiláticas, designadamente repouso no leito (até desaparecimento da cefaleia com um máximo de 48 horas), hidratação forçada (glucose $5 \%$ em soro fisiológico a $125 \mathrm{~mL} / \mathrm{h}$ endovenoso e hidratação por via oral), bebidas à base de cafeína (equivalente a 300-500 mg de cafeína por via oral, 1 a 2 vezes por dia), analgésicos e/ou anti-inflamatórios não esteróides (paracetamol $1 \mathrm{~g}$ de $8 / 8$ horas por via oral e/ou ibuprofeno $400 \mathrm{mg}$ de $8 / 8$ horas por via oral) (Tabela 3).

Quarenta e cinco $(77,6 \%)$ mulheres desenvolveram cefaleia pós-punção (Tabela 4). O início dos sintomas foi maioritariamente $(86,6 \%)$ até às 48 horas após a punção, mas $6,7 \%$ das mulheres desenvolveram cefaleia após 72 horas. A todas as puérperas, incluíndo as 13 que não desenvolveram cefaleia, foi recomendada a ida ao hospital no caso de surgimento tardio de cefaleia. A maior parte $(80,0 \%)$ referiu cefaleia de intensidade moderada. A incidência de CPPD foi semelhante após epidural e CNE (75\% vs $100 \%, p>0,05)$ e não se relacionou com a posição na qual a técnica foi realizada (decúbito lateral esquerdo $77,5 \%$ vs sentada $77,8 \%, p>0,05$ ) ou se o cateter foi colocado no espaço epidural ou intra-tecal $(82,2 \%$ vs $55,6 \%$, $p>0,05)$. Não encontrámos associação entre a CPPD e o tipo de parto (vaginal $73,7 \%$ vs cesariana $85,0 \%, p>0,05$ ).

O tratamento conservador (repouso no leito, hidra-

Tabela 2 - Características da técnica da qual resultou a punção acidental da dura $(n=58)$

\begin{tabular}{llc}
\hline & & PAD \\
$n(\%)$ & $52(89,7)$ \\
Técnica & Epidural & $6(10,3)$ \\
& CNE & $18(31,1)$ \\
Posição & Sentada & $40(69,9)$ \\
& Decúbito lateral esquerdo & $5(8,6)$ \\
Nível vertebral inicial & L2-L3 & $48(82,8)$ \\
& L3-L4 & $5(8,6)$ \\
Local saída LCR & L4-L5 & $34(58,6)$ \\
& Agulha epidural & $8(13,8)$ \\
\hline
\end{tabular}

n: número de doentes; CNE: combinada do neuroeixo; LCR: líquido cefalorraquidiano; PAD: punção acidental da dura.

Tabela 3 - Abordagem após a punção acidental da dura

\begin{tabular}{llc}
\hline & & $\begin{array}{c}\text { PAD detetada e/ou não reconhecida } \\
(\mathrm{n}=42 \text { e/ou } 4)\end{array}$ \\
\hline Cateter epidural lombar & Mesmo espaço & $21(50,0)$ \\
Cateter intra-tecal & Espaço diferente & $9(21,4)$ \\
Abandono técnica loco-regional & & $9(21,4)$ \\
Medidas profiláticas & $3(7,2)$ \\
\hline
\end{tabular}

n: número de doentes; PAD: punção acidental da dura. 
Tabela 4 - Características, fatores de risco e tratamento da cefaleia pós-punção da dura

\begin{tabular}{|c|c|c|c|c|}
\hline \multicolumn{3}{|l|}{ CPPD } & & $p$ \\
\hline \multicolumn{3}{|l|}{$\mathbf{n}$} & \multicolumn{2}{|l|}{$45 / 58(77,6)$} \\
\hline \multirow{3}{*}{ Intensidade } & \multicolumn{2}{|l|}{ Leve } & \multicolumn{2}{|l|}{$5(11,1)$} \\
\hline & \multicolumn{2}{|l|}{ Moderada } & \multicolumn{2}{|l|}{$36(80,0)$} \\
\hline & \multicolumn{2}{|l|}{ Severa } & \multicolumn{2}{|l|}{$4(8,9)$} \\
\hline \multirow{4}{*}{ Tempo de início } & \multicolumn{2}{|l|}{$<24 \mathrm{~h}$} & \multicolumn{2}{|l|}{$16(35,5)$} \\
\hline & \multicolumn{2}{|l|}{$24-48 h$} & \multicolumn{2}{|l|}{$23(51,1)$} \\
\hline & \multicolumn{2}{|l|}{$48-72 h$} & \multicolumn{2}{|l|}{$3(6,7)$} \\
\hline & \multicolumn{2}{|l|}{$>72 \mathrm{~h}$} & \multicolumn{2}{|l|}{$3(6,7)$} \\
\hline \multicolumn{3}{|l|}{ Sintomas associados } & \multicolumn{2}{|l|}{$20 / 45(44,4)$} \\
\hline \multicolumn{3}{|l|}{ Tratamento conservador } & \multicolumn{2}{|l|}{$45(100)$} \\
\hline Técnica & \multicolumn{2}{|l|}{ Epidural vs CNE } & $39(75,0)$ vs $6(100)$ & $>0,05$ \\
\hline Posição & \multicolumn{2}{|c|}{ Decúbito lateral esquerdo vs Sentada } & $31(77,5)$ vs $14(77,8)$ & $>0,05$ \\
\hline Localização do cateter & \multicolumn{2}{|l|}{ Epidural vs Intra-tecal } & $37(82,2)$ vs $5(55,6)$ & $>0,05$ \\
\hline Tipo de parto & \multicolumn{2}{|l|}{ Vaginal vs Cesariana } & $28(73,7)$ vs $17(85,0)$ & $>0,05$ \\
\hline \multicolumn{3}{|c|}{ Instituição medidas profiláticas vs não instituição } & $25(71,4)$ vs $11(72,7)$ & $>0,05$ \\
\hline \multirow{5}{*}{ BPE terapêutico } & \multicolumn{2}{|l|}{ Sim } & \multicolumn{2}{|l|}{$19 / 58(32,8)$} \\
\hline & \multirow{3}{*}{ Timing da realização } & $24-48 h$ & \multicolumn{2}{|l|}{$2(10,5)$} \\
\hline & & $48-72 h$ & \multicolumn{2}{|l|}{$4(21,1)$} \\
\hline & & $>72 \mathrm{~h}$ & \multicolumn{2}{|l|}{$13(68,4)$} \\
\hline & \multicolumn{2}{|l|}{ Repetição BPE } & $3 / 19(15,8)$ & \\
\hline
\end{tabular}

n: número de doentes; CNE: combinada do neuroeixo; CPPD: cefaleia pós-punção acidental da dura; BPE: blood patch epidural.

tação forçada, bebidas com cafeína, analgésicos e/ou anti-inflamatórios não esteróides) foi realizado em todas as doentes que desenvolveram cefaleia $(n=45)$. $O$ blood patch epidural foi considerado como opção terapêutica apenas na persistência de cefaleia por falência do tratamento conservador e foi realizado em 32,8\% (19 em 58) das doentes com um sucesso de $84,2 \%$ na resolução dos sintomas. O BPE foi efetuado após 48 horas do início da sintomatologia na maior parte dos casos (89,5\%). A técnica consistiu na injeção lenta de sangue autólogo no espaço epidural até um volume de $20 \mathrm{~mL}$. A realização de um segundo BPE foi necessária em 15,8\% (3/19) das doentes. O tempo médio global de internamento foi de 4,83 dias (2 - 11 dias, IC 95\%).

\section{DISCUSSÃO}

Nas grávidas submetidas a anestesia epidural, a incidência de punção da dura varia entre 0 a 2,6\%.4,5 No Reino Unido, a taxa descrita de PAD é inferior a 1\%. ${ }^{1,6}$ A população obstétrica tem um risco particularmente aumentado de desenvolver cefaleia após punção inadvertida. Após punção acidental com uma agulha Tuohy $16 \mathrm{G}, 70 \%$ a $80 \%$ das grávidas relatam sintomas relacionados com a baixa pressão do LCR. 1,7 Apesar da utilização de agulhas de calibre 18G diminuir a intensidade da cefaleia, a incidência da cefaleia é semelhante com as agulhas $16 \mathrm{G}$ e $18 \mathrm{G}(70-88 \%$ vs $64 \%){ }^{2}$ A analgesia/anestesia subaracnóideia tem uma incidência de cefaleia reduzida, inferior a $0,5 \%$, devido à utilização de agulhas ponta de lápis de pequeno calibre, 27G. No nosso estudo, a incidência global de PAD resultante de analgesia/ anestesia epidural ou CNE foi de $0,3 \%$ e de CPPD foi de $77,6 \%$, estando de acordo com os dados presentes na literatura.

Após verificação da PAD podem ser adotadas várias atitudes, nomeadamente a re-colocação do cateter epidural no mesmo ou em diferente espaço vertebral, a sua introdução no espaço intra-tecal ou o abandono da técnica regional, optando, por exemplo, por analgesia sistémica. À semelhança do nosso estudo, a re-colocação do cateter epidural num nível vertebral lombar diferente do inicial foi a prática mais frequentemente relatada num questionário efetuado a 160 Anestesiologistas na América do Norte. ${ }^{8}$ Embora tenha ocorrido, ao longo dos anos, um aumento na colocação dos cateteres intra-tecais (CIT), a re-colocação do cateter epidural num espaço vertebral adjacente permanence a opção preferida após punção acidental.9,10

A taxa de colocação do cateter intra-tecal após PAD é muito variável. Estudos efetuados no Reino Unido, Estados Unidos da América e Austrália relataram uma taxa de colocação do CIT de $28 \%, 18,5 \%$ e $35 \%$, respetivamente. , $^{9,11,12}$ 
Em 2011, um questionário a Anestesiologistas do América do Norte relatou uma taxa de apenas $6 \% .^{8}$ A colocação de cateteres intra-tecais tem sido associada à redução na frequência de CPPD. ${ }^{12-14}$ Uma revisão de dez anos num departamento de Anestesiologia Obstétrica resumiu os resultados de vários estudos e concluiu que a incidência de cefaleia foi de $51 \%$ quando o CIT foi colocado e de $66 \%$ quando foi colocado um cateter epidural e a incidência de realização de BPE foi de $33 \%$ e $59 \%$, respetivamente..$^{15}$ Apesar destes resultados a favor da colocação do CIT, esta revisão reuniu dados prospetivos e retrospetivos e muitos dos estudos não eram randomizados nem tinham poder suficiente. Noutro estudo, os autores colocaram e mantiveram o CIT durante 24 horas após a punção acidental. Na sua população obstétrica, a introdução do cateter no espaço subaracnoideu resultou em cefaleia em apenas $6,2 \%$ das doentes, com uma incidência esperada superior a $50 \% .^{13}$ No entanto, a redução na incidência de cefaleia não foi relatada em estudos quando o CIT permanece no local por menos de 24 horas. O mecanismo proposto associado ao benefício da manutenção do cateter no espaço subaracnoideu é a reação ao próprio cateter, com inflamação ou edema, que previne a perda adicional de LCR após remoção do cateter. ${ }^{10}$ Mais recentemente, em 2012, concluiu-se que a conversão para analgesia subaracnóideia após punção acidental não reduziu a incidência de cefaleia nem a necessidade de realização de BPE, mas associou-se ao estabelecimento mais rápido de analgesia do neuroeixo. ${ }^{16}$ No nosso estudo, constatámos que o cateter foi colocado no espaço intra-tecal em $21,4 \%$ dos casos, mas não verificámos diferença na incidência de cefaleia quando comparámos a introdução do CIT com a sua re-colocação no espaço epidural $(55,6$ vs 82,2, $p>0,05)$. Assim sendo, a decisão de colocação do cateter intra-tecal tem de ser ponderada, porque tem a vantagem de proporcionar uma anestesia subaracnóideia rápida e eficaz e de eliminar a possibilidade de outra PAD, mas a desvantagem associada ao seu uso acidental ou infeção. ${ }^{10}$

Existem vários fatores de risco relacionados com o desenvolvimento de cefaleia após punção inadvertida da dura. A escolha da técnica e do tipo e tamanho da agulha para punção lombar são fatores sobre os quais o anestesiologista tem o maior controlo na redução da incidência de CPPD..$^{2,17}$ No nosso estudo, não pudemos retirar ilações fidedignas quanto à incidência de cefaleia quando comparámos a técnica epidural com a técnica CNE $(75,0 \%$ vs $100 \%, p>0,05)$, dado existir uma punção acidental prévia da dura com a agulha de Tuohy nas doentes submetidas a técnica CNE (fator confundidor). Não nos foi possível retirar conclusões quanto à agulha que puncionou acidentalmente a dura, na medida em que foram utilizados o mesmo tipo e calibre em ambas as técnicas (Tuohy 18G).

Vários detalhes relacionados com o procedimento não parecem influenciar a taxa de desenvolvimento de cefaleia, incluindo a posição da doente na altura da punção meníngea. ${ }^{10}$ Não encontrámos diferença na incidência de cefaleia entre a posição de decúbito lateral esquerdo e a posição sentada $(77,5 \%$ vs $77,8 \%, p>0,05)$.

Uma revisão recente observa uma forte associação entre taxas mais baixas de cefaleia em mulheres submetidas a cesariana em comparação com aquelas com parto vaginal. ${ }^{17} \mathrm{~A}$ análise dos dados revelou uma incidência de cefaleia após punção inadvertida de cerca de $11 \%$ em muIheres submetidas a cesariana em comparação com mais de $75 \%$ nas que tiveram parto vaginal, com um dos estudos a encontrar correlação entre o tempo de puxar durante o segundo estádio do trabalho de parto e o desenvolvimento de cefaleia. ${ }^{18,19}$ Noutro trabalho, a incidência de cefaleia no parto vaginal foi superior ( $80 \%$ ) comparativamente com a cesariana (15\%), o que sugeriu que a manobra de valsalva durante o segundo estádio do trabalho de parto pode aumentar a laceração na dura após a punção efetuada pela agulha de Tuohy. ${ }^{20}$ Contudo, são necessários novos estudos para corroborar esta hipótese, na medida em que outras investigações não demonstraram existir associação entre o puxar durante o segundo estádio do trabalho de parto e o aumento na incidência de cefaleia. ${ }^{7,21}$ No nosso estudo, não houve diferença na incidência de CPPD com o tipo de parto, vaginal ou cesariana $(73,7 \%$ vs $85,0 \%, p>$ 0,05).

Após punção acidental da dura, a instituição de medidas profiláticas com o objetivo de reduzir o desenvolvimento de cefaleia ainda é efetuada pelos profissionais, apesar do baixo nível de evidência. ${ }^{8}$ A prescrição de hidratação oral profilática ocorre entre $62 \%$ a $93 \%$, verificando-se, em 2011 , uma taxa de $75 \% .^{7,8,9,11,22}$ O repouso profilático no leito é realizado entre $14 \%$ a $56 \%$ e o incentivo ao consumo de bebidas com cafeína ocorre entre $16 \%$ a $58 \% .^{7,8,9,11,22}$ Embora nenhuma das medidas tenha demonstrado a sua eficácia, estudos recentes em doentes obstétricas ${ }^{8}$ e não obstétricas ${ }^{9}$ mostram que $74 \%$ a $89 \%$ dos profissionais recomendam hidratação oral ou intravenosa agressiva, $48 \%$ a $56 \%$ incentivam ao repouso no leito e $47 \%$ a $58 \%$ prescrevem opióides e fármacos não opióides. ${ }^{23,24} \mathrm{~A}$ terapêutica com cafeína oral ou intravenosa profilática mostrou-se eficaz num pequeno estudo randomizado, com uma redução absoluta de $27 \%$ no desenvolvimento de cefaleia. ${ }^{25}$ No entanto, duas meta-análises publicadas demonstraram que esta intervenção é ineficaz. ${ }^{24,26}$ Uma meta-análise recente concluiu que estudos anteriores não eram randomizados e não tinham poder suficiente, pequenas séries que mostraram benefício nas técnicas estudadas não tinham grupo de controlo e a heterogeneidade dos resultados significa que nenhuma técnica pode ser recomendada como eficaz. ${ }^{24}$ No nosso estudo, foram instituídas medidas profiláticas (repouso no leito, hidratação forçada, bebidas com cafeína, analgésicos e/ou anti-inflamatórios não esteróides) em $76,1 \%$ das puérperas em que ocorreu punção acidental da dura, mas não obtivémos diferença na taxa de incidência de cefaleia entre as puérperas nas quais as medidas profiláticas foram instituídas e naquelas em que essas atitudes não foram prescritas $(71,4 \%$ vs $72.7 \%, p>0,05)$.

Quando se desenvolve cefaleia após punção inadvertida da dura, está preconizada a realização de tratamento 
conservador e/ou de um blood patch epidural com sangue autólogo como primeira abordagem. As medidas conservadoras são semelhantes às instituídas como profilaxia, designadamente a hidratação oral e/ou endovenosa forçada, a administração oral ou endovenosa de cafeína, o repouso no leito e a prescrição de analgésicos, anti-inflamatórios não esteróides e/ou opióides. A instituição de hidratação oral forçada varia entre $86 \%$ a $93 \%,{ }^{8,9,11,22}$ embora existam autores que não a recomendam. ${ }^{27,28}$ As taxas de administração de cafeína variam entre $32 \%$ e $85 \%,,^{8,9,11,22}$ não existindo ainda consenso já que uma revisão sistemática concluiu que a utilização de cafeína não é uma medida eficaz no tratamento de $\mathrm{CPPD}^{29} \mathrm{e}$, mais recentemente, um estudo caso-controlo, afirmou que a cafeína endovenosa pode ser eficaz na redução da taxa e gravidade da cefaleia. ${ }^{30} \mathrm{~A}$ instituição de repouso no leito varia entre $14 \%$ e $71 \%, 8,9,11,22$ não sendo necessário o repouso absoluto para alívio dos sintomas. ${ }^{31,32}$

Após 48 horas de persistência da cefaleia, recomenda-se a realização de blood patch epidural, ${ }^{28}$ que tem provado ser mais eficaz no tratamento da cefaleia do que as medidas conservadoras. ${ }^{33}$ Inicialmente, o BPE foi associado com taxas de sucesso de cerca de 98\%. Estudos posteriores, relataram o alívio completo dos sintomas em $75 \%$ dos doentes, alívio parcial em $18 \%$ e insucesso em $7 \%$, sendo o atraso no tratamento inferior a quatro dias associado a maior taxa de insucesso. ${ }^{34} \mathrm{O}$ mecanismo implicado no alívio da cefaleia após o BPE é a deslocação do LCR a partir do saco tecal lombar devido ao aumento da pressão epidural com um aumento subsequente da pressão intracraniana. ${ }^{2}$ No nosso estudo, verificámos a realização do BPE em 32,8\% das doentes apenas após a falência do tratamento conservador e, na maior parte dos casos $(89,5 \%)$, foi efetuado após 48 horas do início da sintomatologia com uma taxa de sucesso de $84,2 \%$ na resolução dos sintomas. Houve a necessidade de realização de um segundo BPE em 15,8\% (3/19) das doentes, com alívio completo dos sintomas. O tempo médio global de internamento foi de 4,83 dias, superior ao tempo médio habitualmente cumprido num parto sem complicações.

Como limitações ao estudo, apontamos o facto de ser retrospetivo com uma série de variáveis que não controlamos, como, por exemplo, o facto da técnica analgésica/ anestésica ser efetuada por diferentes Anestesiologistas, e o número limitado de casos. A identificação dos casos de punção acidental/cefaleia pós-punção é dependente do seu registo por parte dos Anestesiologistas, motivo pelo qual não existem garantias de identificação de todos os casos. Não nos foi possível calcular se a incidência e a intensidade de CPPD variam com o tipo de agulha escoIhida, pois apenas foi utilizada a agulha Tuohy de calibre
18G. Identificamos um fator confundidor no subgrupo de doentes submetidas à técnica CNE (6 em 58), a existência de duas punções na dura-máter na mesma doente, uma acidental com uma agulha Tuohy de calibre $18 \mathrm{G}$ e outra intencional com uma agulha ponta de lápis de caliber $27 \mathrm{G}$. Este subgrupo de doentes (6 em 58) foi submetido a uma técnica CNE (agulha através de agulha), após a punção acidental da dura com a agulha Tuohy de calibre 18G, pelo que não podemos comparar a real incidência de cefaleia após as duas técnicas.

\section{CONCLUSÕES}

A incidência de punção acidental da dura e de cefaleia pós-punção da dura foi semelhante à descrita na literatura.

A incidência de cefaleia não se relacionou com a posição da doente durante o procedimento ou o tipo de parto. A inserção de cateter subaracnoideu não preveniu o desenvolvimento de cefaleia, contudo não mantivémos o cateter para além das 24 horas.

Após uma punção inadvertida da dura, a re-colocação do cateter epidural num espaço lombar adjacente mantém-se a opção de eleição e a instituição de medidas profiláticas permanece uma prática frequente, apesar da baixa evidência da sua eficácia. Embora atualmente o blood patch epidural seja o tratamento gold standard na CPPD, na nossa população, só foi realizado após falência do tratamento conservador e com uma elevada taxa de sucesso.

Em suma, considerámos que a realização futura de estudos nacionais seria adequada para calcular a incidência e a abordagem desta complicação da anestesia do neuroeixo no nosso país.

\section{PROTECÇÃO DE PESSOAS E ANIMAIS}

Os autores declaram que os procedimentos seguidos estavam de acordo com os regulamentos estabelecidos pelos responsáveis da Comissão de Investigação Clínica e Ética e de acordo com a Declaração de Helsínquia da Associação Médica Mundial.

\section{CONFIDENCIALIDADE DOS DADOS}

Os autores declaram ter seguido os protocolos do seu centro de trabalho acerca da publicação de dados.

\section{CONFLITOS DE INTERESSE}

Os autores declaram não ter quaisquer conflitos de interesse relativamente ao presente artigo.

\section{FONTES DE FINANCIAMENTO}

Este trabalho não recebeu nenhuma contribuição de subsídio ou bolsa.

2015 jun 17] Disponível em: http://www.ihs-classification.org/en/02 klassifikation/03_teil2/07.02.01_nonvascular.html.

4. Reynolds F. Dural puncture and headache. Br Med J. 1993;306:874-6.

5. Turnbull DK, Shepherd DB. Post-dural puncture headache: pathogenesis, prevention and treatment. Br J Anaesth. 2003;91:718-29. 
6. Pan PH, Bogard TD, Owen MD. Incidence and characteristics of failures in obstetric neuraxial analgesia and anesthesia: a retrospective analysis of 19,259 deliveries. Int J Obstet Anesth. 2004;13:227-33.

7. Costigan SN, Sprigge JS. Dural puncture: the patients' perspective. A patient survey of cases at a DGH maternity unit 1983-1993. Acta Anaesthesiol Scand. 1996;40:710-4.

8. Baysinger CL, Pope JE, Lockhart EM, Mercaldo ND. The management of accidental dural puncture and postdural puncture headache: a North American survey. J Clin Anesth. 2011;23:349-60.

9. Harrington BE, Schmitt AM. Meningeal (postdural) puncture headache, unintentional dural puncture, and the epidural blood patch. A national survey of United States practice. Reg Anesth Pain Med. 2009;34:430-7.

10. Harrington BE. Postdural Puncture Headache. Adv Anesth. 2010;28:111 46

11. Baraz R, Collis RE. The management of accidental dural puncture during labour epidural analgesia: a survey of UK practice. Anaesthesia. 2005;60:673-9

12. Newman M, Cyna A. Immediate management of inadvertent dural puncture during insertion of a labour epidural: a survey of Australian obstetric anaesthetists. Anaesth Intensive Care. 2008;36:96-101.

13. Ayad S, Demian Y, Narouz SN, Tetzlaff JE. Subarachnoid catheter placement after wet tap for analgesia in labor: influence on the risk of headache in obstetric patients. Reg Anesth Pain Med. 2003;28:512-5.

14. Warwick WI, Neal JM. Beyond spinal headache: prophylaxis and treatment of low-pressure headache syndromes. Reg Anesth Pain Med. 2007;32:455-61.

15. Van de Velde M, Schepers R, Berends N, Vandermeersch E, De Buck $\mathrm{F}$. Ten years of experience with accidental dural puncture and post-dural puncture headache in a tertiary obstetric anaesthesia department. Int J Obstet Anesth. 2008;17:329-35

16. Russell IF. A prospective controlled study of continuous spinal analgesia versus repeat epidural analgesia after accidental dural puncture in labour. Int J Obstet Anesth. 2012;21:7-16.

17. Gaiser RR. Postdural puncture headache: a headache for the patient and a headache for the anesthesiologist. Curr Opin Anesthesiol. 2013;26:296-303

18. Scavone BM, Wong CA, Sullivan JT, Yaghmour E, Sherwani SS, McCarthy RJ. Efficacy of a prophylactic epidural blood patch in preventing post dural puncture headache in parturients after inadvertent dural puncture. Anesthesiology. 2004;101:1422-7.

19. Angle P, Thompson D, Halpern S, Wilson DB. Second stage pushing correlates with headache after unintentional dural puncture in parturients. Can J Anaesth. 1999;46:861-6.

20. Konrad C, Schupter G, Wietlisbach M, Gerber H. Learning manual skills in anesthesiology: is there a recommended number of cases for anesthetic procedure? Anesth Analg. 1998;86:635-9.

21. Turnbull DK, Shepard DB. Post-dural puncture headache: pathogenesis, prevention and treatment. Br J Anaesth. 2003;91:718-29.

22. Berger CW, Crosby ET, Grodecki W. North American survey of the management of dural puncture occurring during labour epidural analgesia. Can J Anaesth. 1998;45:110-4.

23. Bradbury CL, Singh SI, Badder SR, Wakely LJ, Jones PM. Prevention of post-dural puncture headache in the parturients: a systematic review and meta-analysis. Acta Anaesthsiol Scand. 2013;57:417-30.

24. Apfel CC, Saxena A, Cakmakkaya OS, Gaiser R, George E, Radke O. Prevention of postdural puncture headache after accidental dural puncture: a quantitative systematic review. Br J Anaesth. 2010;105:25563.

25. Yucel A, Ozyalcin S, Talu GK, Yucel EC, Erdine S. Intravenous administration of caffeine sodium benzoate for postdural puncture headache. Reg Anesth Pain Med. 1999;24:51-4

26. Boonmak P, Boonmak S. Epidural blood patching for preventing and treating post-dural puncture headache. Cochrane Database Syst Rev. 2010;1:CD0011791.

27. Dieterich $\mathrm{M}$, Brandt T. Incidence of post-lumbar puncture headache is independent of daily fluid intake. Eur Arch Psychiatry Neurol Sci. 1988;237:194-6.

28. Nguyen DT, Walters RR. Standardizing management of post-dura puncture headache in obstetric patients: a literature review. Open J Anesthesiol. 2014;4:244-53.

29. Halker RB, Demaerschalk BM, Wellik KE, Wingerchuk DM, Rubin DI, Crum BA et al. Caffeine for the prevention and treatment of postdural puncture headache: debunking the myth. Neurologist. 2007;13:323-7.

30. Ragab A, Facharzt KN. Caffeine, is it effective for prevention of postdural puncture headache in young adult patients? Egyptian J Anaesth. 2014;30:181-6.

31. Tejavanija S, Sithinamsuwan $P$, Sithinamsuwan N, Nidhinandana $S$ Suwantemee J. Comparison of prevalence of post-dural puncture headache between six hour-supine recumbence and early ambulation after lumbar puncture in Thai patients: a randomized controlled study. J Med Assoc Thai. 2006;89:814-20.

32. Kim SR, Chae HS, Yoon MJ, Han JH, Cho KJ, Chung SJ. No effect of recumbency duration on the occurrence of post-lumbar puncture headache with a 22G cutting needle. BMC Neurol. 2012;12:1.

33. van Kooten F, Oedit R, Bakker SL, Dippel DW. Epidural blood patch in post dural puncture headache: a randomized, observer-blind, controlled clinical trial. J Neurol Neurosurg Psychiatry. 2008;79:553-8.

34. Safa-Tisseront V, Thormann F, Malassine P, Henry M, Riou B, Coriat $P$, et al. Effectiveness of epidural blood patch in the management of postdural puncture headache. Anesthesiology. 2001;95:334-39. 